\title{
Management of Persistent Vitreous Hemorrhage and Hyphema after Scleral Fixation Sutured Intraocular Lens Implantation with Air-Fluid Exchange-A Case Report
}

\author{
Yu Ti Teng ${ }^{1}$, Chih Hsin Chen ${ }^{2 *}$ \\ ${ }^{1}$ Department of Ophthalmology, National Cheng Kung University, Taiwan \\ ${ }^{2}$ Department of Ophthalmology, Kaohsiung Chang Gung Memorial Hospital and Chang Gung University, Taiwan
}

Submission: April 24, 2017 ; Published: June 07, 2017

*Corresponding author: Chih Hsin Chen, Department of Ophthalmology, Kaohsiung Chang Gung Memorial Hospital, No. 123, Ta-Pei Road, NiaoSung District, Kaohsiung City 833, Taiwan, Tel: 07-7317123 ext.2801; Email: ahsin4491@cgmh.org.tw

Abstract

Purpose: To evaluate the surgical outcome of vitreous hemorrhage, hyphema, and elevated intraocular pressure (IOP) after scleral fixation sutured intraocular lens (IOL) implantation, managed with air-fluid exchange.

Methods: A case report

Results: A 50-year-old female patient presented with traumatic posterior lens dislocation into the vitreous of the right eye and received a trans pars plana vitrectomy and lensectomy combined with trans-scleral sutured IOL implantation. Persistent hyphema (grade III), corneal blood staining, vitreous hemorrhage, and elevated IOP up to $38 \mathrm{~mm} \mathrm{Hg}$ occurred for 1 week after surgery. Air-fluid exchange with $5 \%$ sulfur hexafluoride (SF6) was performed to evacuate the vitreous hemorrhage and hyphema, and to lower the IOP. After the air-fluid exchange procedure, the hyphema and vitreous hemorrhage completely resolved, IOP (15 mm Hg) normalized, and the sutured IOL was well-positioned 3 days later and thereafter. The patient's best-corrected visual acuity improved to 20/100 after 10 days and to 20/20 after 1 month, and remained 20/20 thereafter without recurrent hyphema, vitreous hemorrhage, elevated IOP, or any other adverse events during the 36 months follow-up period.

Conclusion: Hyphema, vitreous hemorrhage, and elevated IOP are possible complications of the implantation of scleral fixation sutured IOL. When surgical intervention is indicated, air-fluid exchange appears to provide an easy, safe, and effective alternative treatment for managing these concomitant conditions.

Keywords: Hyphema; Scleral fixation sutured IOL; Air fluid exchange

\section{Introduction}

Scleral fixation sutured intraocular lens (IOL) is widely used in aphakic eyes without adequate capsular support. However, complications are occasionally reported, including hyphema and vitreous hemorrhage [1]. When hyphema and/ or vitreous hemorrhage occur and cause severe complications such as elevated intraocular pressure (IOP) resulting in optic nerve atrophy, corneal blood staining, and other adverse events, further treatment is necessary. In this case report, we provide a useful surgical method for managing hyphema and vitreous hemorrhage simultaneously without influencing the stability of the sutured IOL and with a satisfactory visual outcome.

\section{Case report}

A 50 year old, healthy female patient visited our clinic with the complaint of significantly impaired vision in her right eye. She reported experiencing blunt eye trauma caused by a solid object accidentally striking her right eye 2 months prior and having progressively deteriorating vision since. Moreover, over the past week, a dramatic decrease in visual acuity in the injured right eye forced her to seek out our help. During the first visit to our clinic, the patient's best corrected visual acuity (BCVA) was only "counting fingers" in her injured right eye and 20/20 
in her healthy left eye. Ophthalmologic examinations showed a dense nuclear sclerotic lens posteriorly dislocated into the vitreous. After discussing the advantages and disadvantages of 2 applicable surgical methods (anterior chamber IOL implantation or scleral fixation sutured IOL implantation), the patient opted to receive trans pars plana vitrectomy and lensectomy combined with trans-scleral fixation sutured IOL implantation.

The surgical procedures were all performed smoothly except for mild vitreous hemorrhage that occurred when the long needle with 10-0 Prolene passed through the scleral area ( $1.5 \mathrm{~mm}$ posterior to the limbus). After the surgery, hyphema (grade III-more than one half of the anterior chamber filled with blood), moderately dense vitreous hemorrhage, corneal blood staining, and elevated IOP up to $38 \mathrm{~mm} \mathrm{Hg}$ (despite using maximal IOP-lowering medications) persisted for 1 week. Vision in the operated eye dropped to only "hand motion". To prevent the possible irreversible complications of hyphema, corneal blood staining, and elevated IOP-induced optic atrophy, we performed air-fluid exchange with 5\% sulfur hexafluoride (SF6) to evacuate the vitreous hemorrhage and hyphema and to possibly reduce the concomitant IOP. Ten days later, the SF6 gas disappeared and no vitreous hemorrhage or hyphema recurred, the IOP normalized (15 $\mathrm{mm} \mathrm{Hg}$ ), and the patient's BCVA improved to $20 / 100$. Moreover, the BCVA in the operated eye improved to 20/20 after 1 month and was maintained thereafter; meanwhile, the sutured IOL remained well centered and no adverse events occurred during the 36 months follow-up period.

\section{Discussion}

Hyphema is the accumulation of blood in the anterior chamber of the eye, and the etiology of this condition includes eye trauma, intraocular surgery, spontaneous bleeding, or using substances that affect platelet or fibrin function [1]. Although hyphema alone rarely causes permanent vision loss, some complications such as secondary hemorrhage and increased IOP resulting in optic atrophy or corneal blood staining may seriously affect vision. Therefore, different studies offer various recommendations for the treatment of hyphema, including supportive care, pharmacologic therapy, bed rest versus ambulation, admission or outpatient management, eye patching, and even surgical intervention [2-4]. However, the gold standard of management for hyphema remains controversial.

Generally, there is consensus that surgical intervention is reserved for following conditions:

a. Uncontrolled IOP and risk of optic atrophy or central retinal artery occlusion

b. Presentation or risk of corneal blood staining

c. Risk of synechia formation

d. Detection of rebleeding hyphema $[2,3]$.

When surgical intervention is indicated, several methods are available, including paracentesis and anterior chamber irrigation, automated hyphectomy, and blood clot expression or irrigation. However, in our case, hyphema and vitreous hemorrhage coexisted and were difficult to distinguish because the anterior and posterior chambers were communicated; therefore, it may not have been sufficient to treat this case with only anterior chamber irrigation. Nevertheless, air-fluid exchange is an alternative treatment for repeat vitrectomy in patients with post vitrectomy diabetic vitreous hemorrhage $[5,6]$.

Some reports have demonstrated the efficacy and low complication risk of air-fluid exchange in managing post vitrectomy vitreous hemorrhage $[5,6]$. Therefore, we applied this concept to the present case. Air-fluid exchange with shortduration gas (5\% SF6) was performed to simultaneously evacuate the concomitant hyphema and vitreous hemorrhage. The BCVA in the affected eye improved from hand motion to $20 / 100$ at 10 days after the operation, and further improved to $20 / 20$ at 1 month, and was sustained thereafter. In the next 36 months follow-up period, the sutured IOL remained well centered without recurrent hyphema, vitreous hemorrhage, elevated IOP, or any other adverse events.

Hyphema is a possible complication of implantation of scleral fixation sutured IOL. Although such complications have been reported in some studies [7,8], they either rarely mention the management of the hyphema or only conservative treatments were used. When surgical intervention for hyphema is indicated, our case report suggests that air-fluid exchange provides an easy, safe, and effective alternative for managing this concomitant condition. With this surgical method, vision improved significantly and the structure and stability of the scleral fixation sutured IOL was not affected. However, a larger number of cases and longer follow-up periods are required to evaluate the efficacy and safety of air-fluid exchange treatment for concomitant hyphema and vitreous hemorrhage after scleral sutured IOL implantation.

\section{Conclusion}

Scleral fixation sutured IOL implantation procedures may cause complications, including hyphema and vitreous hemorrhage. Although conservative treatments are applicable in most cases, surgical intervention may be indicated for some conditions, such as increased IOP resulting in optic atrophy or corneal blood staining. When surgery is necessary, air-fluid exchange may be considered as an alternative treatment as it is a useful, easy, and safe way to wash out hyphema and vitreous hemorrhage simultaneously without influencing the stability of the sutured IOL and with a satisfying visual outcome.

\section{References}

1. McAllister AS, Hirst LW (2011) Visual outcomes and complications of scleral-fixated posterior chamber intraocular lenses. J Cataract Refract Surg 37(7): 1263-1269.

2. William Walton, Stanley Von Hagen, Ruben Grigorian, Marco Zarbin (2002) Management of Traumatic Hyphema. Surv Ophthalmol 47(4): 297-334. 
3. Brandt MT, Haug RH (2001) Traumatic Hyphema: A Comprehensive Review. J Oral Maxillofac Surg 59(12): 1462-1470.

4. Gharaibeh A, Savage HI, Scherer RW, Goldberg MF, Lindsley K (2011) Medical interventions for traumatic hyphema. Cochrane Database Syst Rev 19(1): CD005431.

5. Martin DF, McCuen BW (1992) Efficacy of fluid-air exchange for post vitrectomy diabetic vitreous hemorrhage. Am J Ophthalmol 114(4): 457-463.

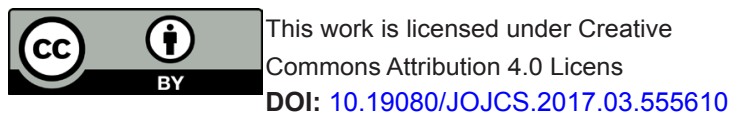

6. Han DP, Murphy ML, Mieler WF, Abrams GW (1991) Outpatient fluidair exchange for severe postvitrectomy diabetic vitreous hemorrhage. Long-term results and complications. Retina 11(3): 309-314

7. Kjeka O, Bohnstedt J, Meberg K, Seland JH (2008) Implantation of scleral-fixated posterior chamber intraocular lenses in adults. Acta Ophthalmol 86(5): 537-542.

8. Monteiro M, Marinho A, Borges S, Ribeiro L, Correia C (2007) Scleral fixation in eyes with loss of capsule or zonule support. J Cataract Refract Surg 33(4): 573-576.

\section{Your next submission with Juniper Publishers will reach you the below assets}

- Quality Editorial service

- Swift Peer Review

- Reprints availability

- E-prints Service

- Manuscript Podcast for convenient understanding

- Global attainment for your research

- Manuscript accessibility in different formats

( Pdf, E-pub, Full Text, Audio)

- Unceasing customer service

Track the below URL for one-step submission https://juniperpublishers.com/online-submission.php 